\title{
Estimating the effective conductivity for ellipse-inclusion model with Kapitza thermal resistance
}

\author{
Van-Luat Nguyen ${ }^{*}$ (DD \\ Faculty of Mechanical Engineering, Hanoi University of Industry, 298 Cau Dien Street, Bac Tu Liem District, Hanoi, Vietnam
}

Received: 4 March 2021 / Accepted: 14 July 2021

\begin{abstract}
The ellipse assemblage model with imperfect interface has quite complex microstructure, that can be considered an extension of the circle assemblage model with imperfect interfaces. The paper introduces an approximate method for computing the effective conductivity of isotropic composites with imperfect interfaces in two-dimensional space. Based on the coated-ellipse assemblage model and the equivalent inclusion approximation, one can determine the effective thermal conductivity of the composites. The polarization approximation is given in an explicit form (PEK) and this method will be applied to calculate the effective conductivity of the composite with Kapitza thermal resistance model. The PEK result will have compared with the Fast Fourier Transform (FFT) simulation and Hashin-strikman bounds (HS).
\end{abstract}

Keywords: Effective conductivity / homogenization / imperfect interface /

elliptical Kapitza thermal resistance

\section{Introduction}

Due to limited available information about the materials as well as the complexity of microstructure, the determination of macroscopic moduli of composite materials is not simple. Especially composite materials with imperfect contact surfaces, this model needs to have its own approach solution, that will be presented in this work. Determination of macroscopic properties of composite materials from the properties of the component materials and their microstructure (homogenization) is the subject of many studies in recent decades. There are only a few cases where macroscopic properties are determined exactly. The solution of the problem of homogenization can be divided into the following directions: The first one is to construct the upper and lower bounds on the effective properties based on variational principles [1-4], the second one is to develop the approximate methods using Eshelby's solution [5] such as [6-10]. Besides, with the fast development of computer science, the numerical methods [10-15] have been applied in mechanics of materials and effective in solving complex problems. The objective of this research part is to present the methods (approximateanalytic and numerical simulation), which determine macroscopic properties of composite materials with

\footnotetext{
* e-mail: luatnv1980@gmail.com
}

complex microstructures. With complex inclusion such as coated-inclusions or those with imperfect interfaces, the fields inside and around the inclusion are complex, making more difficult the development of approximate models. Benveniste et al. [16] has developed two models of thin interface in the problem of thermal conductivity and elasticity, the model allows determining the fields of adjacent phases without these fields of interphase itself. Le Quang et al. [17] have been derived the closed-form expressions for the effective conductivity of anisotropic multiphase composites with imperfect interfaces by using the Eshelby's formalism and dilute solution results. Yvonnet et al. [11] have used XFEM method to the thermal conductivity of the material with imperfect interfaces. Monchiet [15] has developed algorithms based on XFFT to simulate discontinuous phenomenon of temperature between the phases (phenomenon Kapitza). Nguyen [12] has developed a simple approach to approximate macroscopic properties of the material in this case by replacing the coated-ellipse inclusion by a homogeneous equivalent inclusion. Many authors have mentioned the phenomenon of imperfect interfaces but most applications of these studies are based on the solution of circle-inclusion models. This work focuses on the conductivity of ellipse assemblage model with imperfect interfaces, in which the solution for this problem is based on polarization approximation (PEK) with elliptical Kapitza thermal resistance. 


\section{The ellipse inclusion with interfacial thermal resistance}

We consider the two-component coated ellipsoid assemblage with thin coating (volume fractions $v_{2} \ll 1, v_{1}+v_{2}=1$ ), in which the ellipse made material-1 is the inclusion the ellipse shells made material-2 is the matrix. In the imperfect interfaces model with the infinitely thin coating thickness $h$, $\left(h \sum_{i=1}^{d} \frac{1}{a_{i}} \ll 1\right)$. Because elliptical inclusions of phase 1 coated by the infinitely thin shells, we can consider a uniform thickness interphase.

We have

$$
\begin{gathered}
\frac{v_{1}+v_{2}}{v_{1}}=\frac{1}{v_{1}}=\frac{\pi \cdot \prod_{i=1}^{d}\left(a_{i}+h\right)}{\pi \cdot \prod_{i=1}^{d} a_{i}}=1+\sum_{i=1}^{d} \frac{h}{a_{i}}+O\left(h^{2}\right) \\
\frac{1}{v_{1}}=\frac{1}{1-v_{2}}=1+v_{2}+O\left(v_{2}^{2}\right) \\
\Rightarrow v_{2}=\sum_{i=1}^{d} \frac{h}{a_{i}}
\end{gathered}
$$

where $a_{1}, a_{2}, a_{3}$ are the principal radii of the ellipsoid in the direction of the axes $x_{1}, x_{2}, x_{3}$.

In the case of two component matrix composites with inclusions of ellipsoidal forms, in which $v_{I}, c_{I}$ are the volume fraction and conductivity of the inclusion; $v_{M}, c_{M}$ are the volume fraction and conductivity of the matrix. The expression of the effective conductivity for the dilute suspension of randomly oriented ellipsoids (hence the effective medium is isotropic) can be presented as $\left(v_{I} \ll 1, v_{I}+v_{M}=1\right)[5]$

$$
C^{e f f}=c_{M}+v_{I}\left(c_{I}-c_{M}\right) D\left(c_{I}, c_{M}\right)+O\left(v_{I}^{2}\right)
$$

where $D$ is the respective inclusion-function. In the case of two-dimensional space $(d=2)$ with the same aspect ratio $r=a_{2} / a_{1}$ in a continuous matrix, one has

$$
D\left(c_{I}, c_{M}\right)=\frac{c_{M}\left(c_{I}+c_{M}\right)(1+r)^{2}}{2\left(c_{I}+r c_{M}\right)\left(r c_{I}+c_{M}\right)} .
$$

On the other side, we used the polarization approximation for two-component, the effective conductivity $C^{\text {eff }}$ has form

$$
\begin{aligned}
C^{e f f}=P_{C}\left(c_{*}\right)= & \left(\frac{v_{I}}{c_{I}+(d-1) c_{*}}+\frac{v_{M}}{c_{M}+(d-1) c_{*}}\right)^{-1} \\
& -(d-1) c_{*}
\end{aligned}
$$

where the reference parameter $c_{*}$ should be determined from a reference dilute solution result, or reference effective conductivity of the composite at certain finite volume proportions of the components. Note that, function $P_{C}\left(c_{*}\right)$ is a positive monotonous-increasing function of the positive parameter $c_{*}$.
The polarization approximation $P_{C}\left(c_{*}\right)$ would obey Hashin-Strikman bounds [1]:

$$
P_{C}\left(c_{\min }\right) \leq P_{C}\left(c_{*}\right) \leq P_{C}\left(c_{\max }\right)
$$

the respective reference parameter $c *$ should lie within the limits

$c_{\min } \leq c_{*} \leq c_{\max }, c_{\min }=\min \left\{c_{I}, c_{M}\right\}, c_{\max }=\max \left\{c_{I}, c_{M}\right\}$

The polarization approximation (4) using dilute solution reference (2) or equalizing (4) and (2), one finds the reference parameter $c_{*}$ in the two-dimensional space $(d=2)$

$$
c_{*}=\frac{D\left(c_{I}, c_{M}\right) c_{I}-c_{M}}{1-D\left(c_{I}, c_{M}\right)}
$$

Substituting (7) into (4) after some manipulation, one obtains

$$
C^{e f f}=\frac{c_{M} v_{M}+c_{I} v_{I} D\left(c_{I}, c_{M}\right)}{v_{M}+v_{I} D\left(c_{I}, c_{M}\right)} .
$$

The application (8) for coated-ellipse inclusions, consider the ellipse made of material-1 are embedded in the ellipse shell of material-2, in which the ellipse made material- 1 is the inclusion with conductivity $c_{I}=c_{1}$, the ellipse shell made material-2 is the matrix with conductivity $c_{M}=c_{2}$.

In the context of thermal conduction with the imperfect interface models, the most widely used ones are the Kapitza's interface thermal resistance model, called also lowly conducting (LC) interface model, and the highly conducting $(\mathrm{HC}$ ) interface model. In this work, we focus on the lowly conducting imperfect interface model $[17,18]$, it is assumed that

$$
c_{2}=\frac{h}{\alpha}, h \sum_{i=1}^{d} \frac{1}{a_{i}} \rightarrow 0
$$

where $\alpha$ is called the Kapitza thermal resistance.

Based on (9), (8) and (3), one derives the asymptotic expression of the effective conductivity $c_{1 L}$ of the assemblage of ellipse inclusions of phase- 1 coated by the infinitely thin shells (phase-2) with thermal resistance $\alpha$ (Fig. 1)

$$
\begin{aligned}
c_{1 L}= & \frac{c_{2} v_{2}+c_{1} v_{1} D\left(c_{1}, c_{2}\right)}{v_{2}+v_{1} D\left(c_{1}, c_{2}\right)}=\frac{c_{1}+\frac{c_{2} v_{2}}{v_{1} D\left(c_{1}, c_{2}\right)}}{1+\frac{v_{2}}{v_{1} D\left(c_{1}, c_{2}\right)}} \\
= & \frac{c_{1}+\frac{2 v_{2}\left(c_{1}+r c_{2}\right)\left(c_{2}+r c_{1}\right)}{v_{1}\left(c_{1}+c_{2}\right)(1+r)^{2}}}{1+\frac{2 v_{2}}{c_{2}} \frac{\left(c_{1}+r c_{2}\right)\left(c_{2}+r c_{1}\right)}{v_{1}\left(c_{1}+c_{2}\right)(1+r)^{2}}} .
\end{aligned}
$$




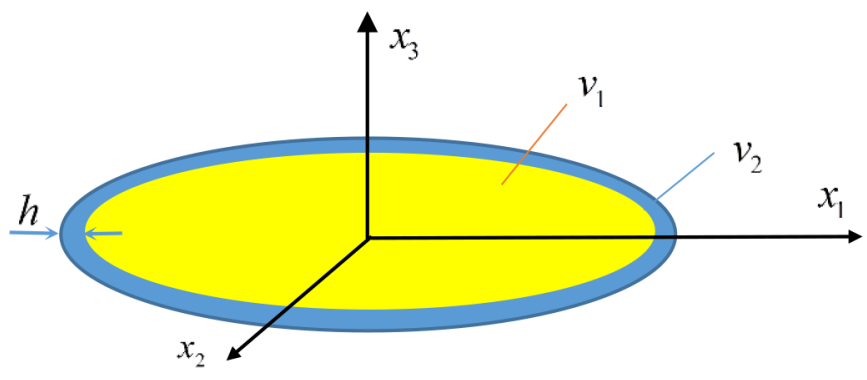

Fig. 1. A single Coated ellipsoid.
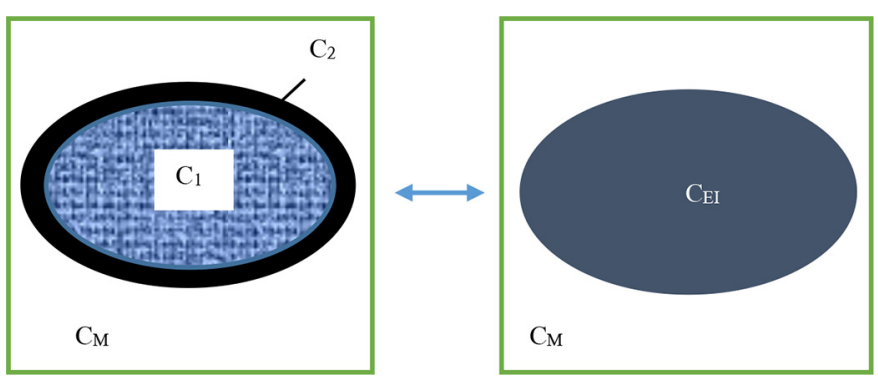

Fig. 2. Unit cell: Coated ellipse (left), equivalent inclusion (right).

Substituting (1) and (9) into (10) (in the limit $\left.h \rightarrow 0, v_{1}=1\right)$ we come to

$$
\begin{gathered}
c_{1 L}=\frac{c_{1}+\frac{2 \sum_{i=1}^{d} \frac{h}{a_{i}}\left(c_{1}+r \frac{h}{\alpha}\right)\left(\frac{h}{\alpha}+r c_{1}\right)}{\left(c_{1}+\frac{h}{\alpha}\right)(1+r)^{2}}}{1+\frac{2 \sum_{i=1}^{d} \frac{\alpha}{a_{i}}\left(c_{1}+r \frac{h}{\alpha}\right)\left(\frac{h}{\alpha}+r c_{1}\right)}{\left(c_{1}+\frac{h}{\alpha}\right)(1+r)^{2}}} \\
\simeq \frac{c_{1}}{1+2 \alpha c_{1} \frac{r}{(1+r)^{2}} \sum_{i=1}^{d} \frac{1}{a_{i}}} .
\end{gathered}
$$

Especially, when $r=1, a_{1}=a_{2}=R, d=2$, we derive the asymptotic expression of the effective conductivity $c_{1 L}$ of the assemblage of circular inclusions, that has been presented in [19]

$$
c_{1 L}=\frac{c_{1}}{1+\frac{\alpha c_{1}}{R}} .
$$

For the coated ellipse assemblage model, if these coated inclusions are embedded in the elliptic shells of the matrix phase $\mathrm{M}$, one obtains the effective conductivity of the elliptic assemblage with interfacial thermal resistance $\alpha$ (in the limit $h \rightarrow 0$ ) between the inclusion 1 and matrix $\mathrm{M}$ components by replacing $c_{E I}$ for $c_{I}$ in (4), (7) and (8) (Fig. 2). The two-phase coated inclusions with imperfect interface are substituted by equivalent one-phase inclusions $c_{E I}=c_{1 L}$ (12), from (7) we have

$$
c_{* E I}=\frac{D\left(c_{1 L}, c_{M}\right) c_{1 L}-c_{M}}{1-D\left(c_{1 L}, c_{M}\right)}
$$

The polarization approximation has the form (PEK)

$$
C^{e f f}=P_{C}\left(c_{* E I}\right)=\left(\frac{v_{1}}{c_{1 L}+c_{* E I}}+\frac{v_{M}}{c_{M}+c_{* E I}}\right)^{-1}-c_{* E I} .
$$

The polarization approximation (14) contains all the components of the coated-ellipse assemblage model with imperfect interface such as $c_{1}, c_{M}, \alpha, a_{1}, a_{2}$. So, one can apply the simple polarization approximation (14) to obtain an estimate for the effective conductivity of the original elliptic inclusion with imperfect interface.

\section{FFT simulation for composite materials with elliptical Kapitza thermal resistance}

Transverse isotropic unidirectional fiber-reinforced composite is well presented by polydispersed coated ellipse cylinders, whose phase boundaries are cylindrical ellipse surfaces, with generators (in $x_{3}$-direction) orthogonal to the plane of isotropy (Fig. 1). Consider a composite reinforced by fibers aligned in the direction $x_{3}$. The ellipse inclusions are periodically distributed in the directions $x_{1}$ and $x_{2}$. The contact between inclusion and matrix is the imperfect interface of Kapitza's type. This problem can be modelled as a composite of three-component: Inclusions $\left(v_{1}, c_{1}\right)$, matrix $\left(v_{M}, c_{M}\right)$ and interphase with volume fraction $v_{2}$, conductivity $c_{2}=h / \alpha$ (Fig. 2). By substitution scheme, one can consider the two-component of coated-ellipse with perfect interface in which the conductivity of equivalent inclusion is determined by (11)

$$
c_{E I}=c_{1 L}=\frac{c_{1}}{1+2 \alpha c_{1} \frac{r}{(1+r)^{2}} \sum_{i=1}^{d} \frac{1}{a_{i}}} .
$$

The main content of the FFT method is based on the known equations, equilibrium conditions and the Fourier transform of the gradient $\mathbf{E}(x)$, flux field $\mathbf{J}(x)$, the second order local conductivity tensor of the heterogeneous medium $\mathbf{C}(x)$ to establish Lippmann-Schwinger integral equations for heterogeneous material problem and use of the Green periodic operator.

The algorithm for determining the effective conductivity of two-phase periodic materials based on the Fourier transform method (FFT) has been introduced in the literature $[12,14,19]$. In the next section, this method will be applied to calculate the effective conductivity of the composite with Kapitza thermal resistance. 

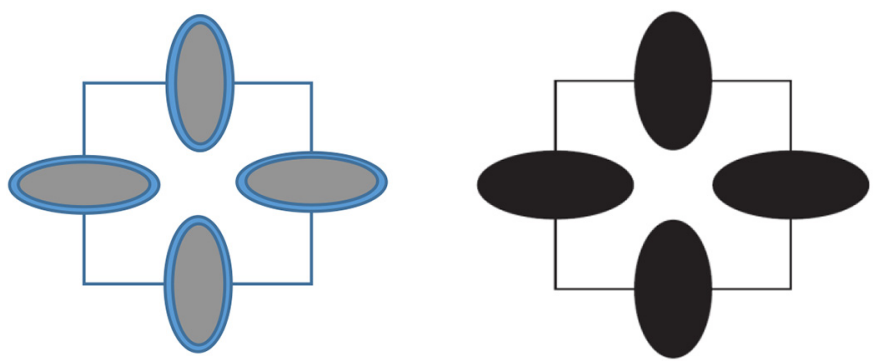

Fig. 3. Unit cell with square ellipse inclusion (left), equivalent inclusion (right).

Table 1. Comparison of results ( $C^{\text {eff }}$ ) of FFT and PEK for square model, $\alpha=1, a_{1}=0.25$.

\begin{tabular}{|c|c|c|c|c|c|c|c|c|c|}
\hline \multicolumn{6}{|c|}{$c_{M}=1, c_{1}=10$} & \multicolumn{4}{|c|}{$c_{M}=1, c_{1}=100$} \\
\hline$r$ & $v_{1}$ & HSL & FFT & PEK & $\mathrm{HSU}$ & HSL & $\mathrm{FFT}$ & PEK & $\mathrm{HSU}$ \\
\hline 0.1 & 0.0393 & 0.8737 & 0.9089 & 0.9091 & 0.9420 & 0.8751 & 0.9096 & 0.9097 & 0.9421 \\
\hline 0.2 & 0.0785 & 0.7881 & 0.8574 & 0.8599 & 0.8898 & 0.7904 & 0.8582 & 0.8607 & 0.8902 \\
\hline 0.3 & 0.1178 & 0.7259 & 0.8174 & 0.8206 & 0.8428 & 0.7289 & 0.8184 & 0.8216 & 0.8435 \\
\hline 0.4 & 0.1571 & 0.6784 & 0.7822 & 0.7853 & 0.8002 & 0.6821 & 0.7835 & 0.7865 & 0.8012 \\
\hline 0.5 & 0.1963 & 0.6409 & 0.7499 & 0.7524 & 0.7616 & 0.6451 & 0.7514 & 0.7539 & 0.7630 \\
\hline 0.6 & 0.2356 & 0.6104 & 0.7195 & 0.7214 & 0.7266 & 0.6151 & 0.7214 & 0.7232 & 0.7283 \\
\hline 0.7 & 0.2749 & 0.5851 & 0.6909 & 0.6921 & 0.6964 & 0.5902 & 0.6932 & 0.6943 & 0.6968 \\
\hline 0.8 & 0.3142 & 0.5637 & 0.6639 & 0.6645 & 0.6655 & 0.5693 & 0.6665 & 0.6672 & 0.6681 \\
\hline 0.9 & 0.3534 & 0.5453 & 0.6383 & 0.6387 & 0.6389 & 0.5514 & 0.6414 & 0.6418 & 0.6420 \\
\hline 1 & 0.3927 & 0.5293 & 0.6140 & 0.6146 & 0.6146 & 0.5359 & 0.6177 & 0.6182 & 0.6182 \\
\hline
\end{tabular}

Table 2. Comparison of results ( $C^{\text {eff }}$ ) of FFT and PEK for square model, $\alpha=0.2, a_{1}=0.25$.

\begin{tabular}{|c|c|c|c|c|c|c|c|c|c|}
\hline \multicolumn{6}{|c|}{$c_{M}=1, c_{1}=10$} & \multicolumn{4}{|c|}{$c_{M}=1, c_{1}=100$} \\
\hline$\underline{r}$ & $v_{1}$ & HSL & FFT & PEK & $\mathrm{HSU}$ & HS & FFT & PEK & $\mathrm{HSU}$ \\
\hline 0.1 & 0.0393 & 0.9823 & 0.9826 & 0.9826 & 0.9831 & 0.9848 & 0.9850 & 0.9850 & 0.9853 \\
\hline 0.2 & 0.0785 & 0.9716 & 0.9721 & 0.9721 & 0.9724 & 0.9768 & 0.9771 & 0.9770 & 0.9772 \\
\hline 0.3 & 0.1178 & 0.9665 & 0.9670 & 0.9669 & 0.9671 & 0.9747 & 0.9749 & 0.9749 & 0.9750 \\
\hline 0.4 & 0.1571 & 0.9662 & 0.9665 & 0.9665 & 0.9666 & 0.9778 & 0.9779 & 0.9779 & 0.9779 \\
\hline 0.5 & 0.1963 & 0.9701 & 0.9702 & 0.9702 & 0.9702 & 0.9856 & 0.9856 & 0.9856 & 0.9856 \\
\hline 0.6 & 0.2356 & 0.9778 & 0.9778 & 0.9778 & 0.9778 & 0.9977 & 0.9977 & 0.9977 & 0.9977 \\
\hline 0.7 & 0.2749 & 0.9890 & 0.9890 & 0.9890 & 0.9890 & 1.0139 & 1.0139 & 1.0139 & 1.0139 \\
\hline 0.8 & 0.3142 & 0.0035 & 1.0035 & 1.0035 & 1.0035 & 1.0340 & 1.0340 & 1.0340 & 1.0341 \\
\hline 0.9 & 0.3534 & 1.0213 & 1.0213 & 1.0213 & 1.0213 & 1.0581 & 1.0581 & 1.0581 & 1.0583 \\
\hline 1 & 0.3927 & 1.0422 & 1.0422 & 1.0422 & 1.0423 & 1.0860 & 1.0860 & 1.0860 & 1.0866 \\
\hline
\end{tabular}

\section{Application and comparison}

For numerical FFT illustrations, consider periodic models: Square model (Fig. 3). We take $c_{M}=1$, the inclusion with $c_{1}>c_{M}, c_{1}=10$ or $c_{1}=100, h=0.0001, c_{2}=h / \alpha$, thermal resistance $\alpha=0.05 ; \alpha=0.2 ; \alpha=1$. Consider a unit cell having the dimension $L=1$ along each space directions containing inclusion with the dimensionless, ratio $r=a_{2} / a_{1}$ varies from 0.1 to 1 , with non-overlapping condition of ellipse inclusions, we assumed that $a_{1}=0.25$ for square model (corresponding volume fraction from 0.0393 to 0.3927).

The results of calculations are reported in Tables 1-3 and Figures 4-9. From all tables and figures, we can see that: the FFT simulation and PEK approximation are coincided and always obey HS bounds (those includes upper bound denoted by HSU and lower bound denoted HSL), one also sees the dependence of the effective 
Table 3. Comparison of results ( $C^{\text {eff }}$ ) of FFT and PEK for square model, $\alpha=0.05, a_{1}=0.25$.

\begin{tabular}{|c|c|c|c|c|c|c|c|c|c|}
\hline \multicolumn{6}{|c|}{$c_{M}=1, c_{1}=10$} & \multicolumn{4}{|c|}{$c_{M}=1, c_{1}=100$} \\
\hline$r$ & $v_{1}$ & HSL & FFT & PEK & $\mathrm{HSU}$ & HSL-EI & FFT & PEK & $\mathrm{HSU}$ \\
\hline 0.1 & 0.0393 & 1.0292 & 1.0324 & 1.0320 & 1.0336 & 1.0365 & 1.0429 & 1.0422 & 1.0458 \\
\hline 0.2 & 0.0785 & 1.0641 & 1.0694 & 1.0686 & 1.0753 & 1.0799 & 1.0904 & 1.0889 & 1.1036 \\
\hline 0.3 & 0.1178 & 1.1043 & 1.1108 & 1.1095 & 1.1248 & 1.1299 & 1.1426 & 1.1402 & 1.1737 \\
\hline 0.4 & 0.1571 & 1.1497 & 1.1565 & 1.1548 & 1.1821 & 1.1867 & 1.1998 & 1.1965 & 1.2566 \\
\hline 0.5 & 0.1963 & 1.2002 & 1.2066 & 1.2045 & 1.2471 & 1.2503 & 1.2626 & 1.2587 & 1.3527 \\
\hline 0.6 & 0.2356 & 1.2559 & 1.2614 & 1.2592 & 1.3199 & 1.3212 & 1.3317 & 1.3275 & 1.4628 \\
\hline 0.7 & 0.2749 & 1.3171 & 1.3214 & 1.3191 & 1.4004 & 1.3998 & 1.4081 & 1.4038 & 1.5876 \\
\hline 0.8 & 0.3142 & 1.3839 & 1.3873 & 1.3849 & 1.4889 & 1.4868 & 1.4932 & 1.4888 & 1.7277 \\
\hline 0.9 & 0.3534 & 1.4568 & 1.4597 & 1.4571 & 1.5855 & 1.5830 & 1.5886 & 1.5836 & 1.8841 \\
\hline 1 & 0.3927 & 1.5363 & 1.5398 & 1.5363 & 1.6905 & 1.6896 & 1.6965 & 1.6896 & 2.0579 \\
\hline
\end{tabular}

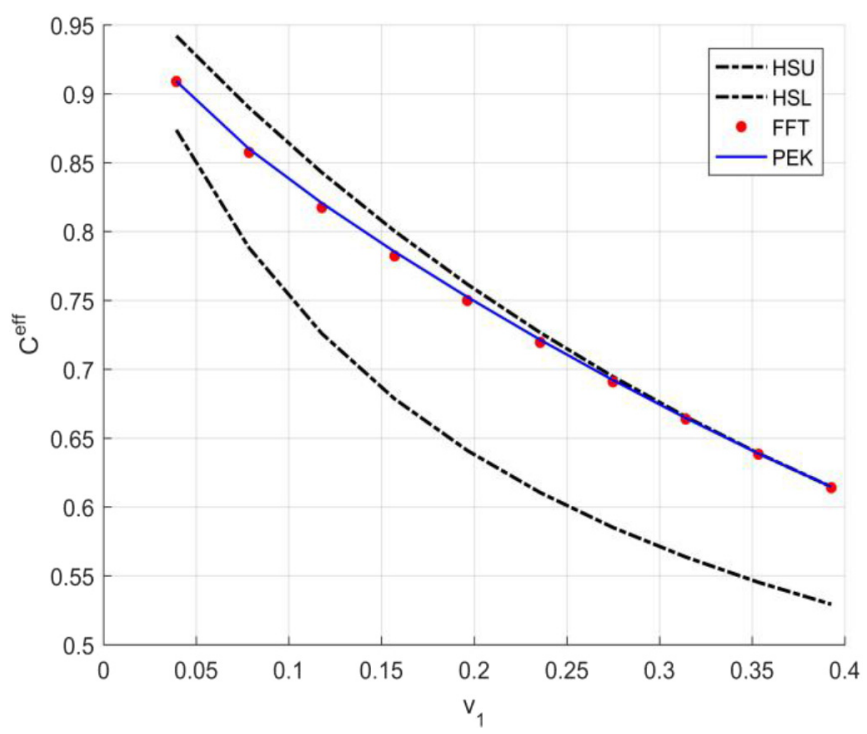

Fig. 4. Effective conductivity of square model with imperfect interface of ellipse Kapitza's type, $\alpha=1,{ }^{c_{1}} /{ }_{c_{M}}=10$.

conductivity on the Kapitza thermal resistance. When $\alpha=1$, volume fraction of inclusion increases but effective conductivity decreases. $\alpha=0.2$ one observes a slight decrease then increase. $\alpha=0.05$ two curves increase. In all cases, when conductivity of the inclusion increases 10 times then effective conductivity increases only slightly. This shows the dominance of the thermal resistance coefficient for the macroscopic conductivity.

The FFT results in a special case $r=1, a_{1}=a_{2}=R$ which were compared with results of Monchiet [15] for square model with one circle inclusion in the unit cell (Fig. 2), the unit cell having the dimension $L=1$ along each space directions containing inclusion with the dimensionless radius $R$ varies from 0.1 to 0.5 . The conductivity of the equivalent inclusion is determined from (13). The result of comparison is reported in Table 4, those are completely close.

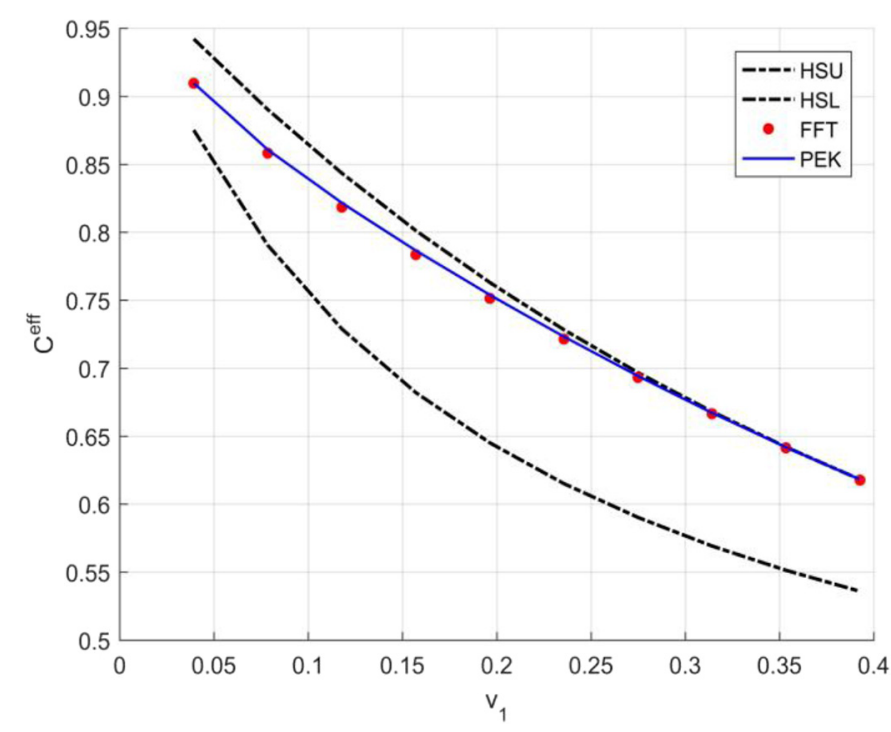

Fig. 5. Effective conductivity of square model with imperfect interface of ellipse Kapitza's type, $\alpha=1,{ }^{c_{1}} /{ }_{c_{M}}=100$.

\section{Conclusion}

The paper has presented a solution for effective conductivity of the coated-ellipse assemblage model with imperfect interfaces, the effective conductivity of isotropic multiphase composites with imperfect interfaces can be determined with two different approaches. The first approach is based on the model of coated-ellipse inclusion with thin coating, the PEK expression for the effective conductivity have been derived by using the Eshelby's formalism and dilute solution results combined with polarization approximation. The second approach is based on the FFT simulation combining equivalent inclusion approach. Using different approaches, polarization approximation and FFT simulation have been proposed to estimate the effective conductivity of isotropic material composites with 


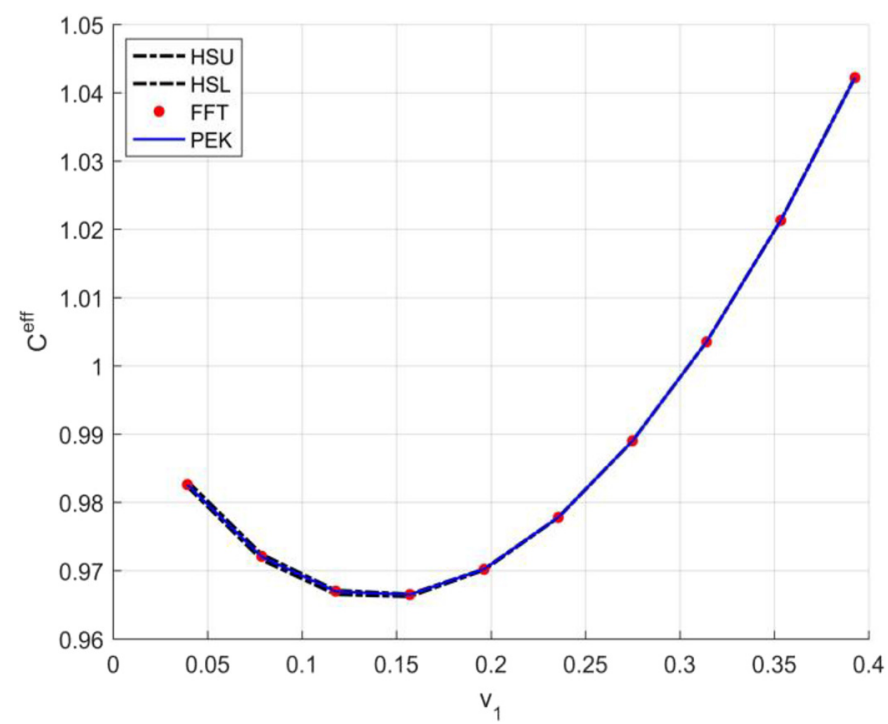

Fig. 6. Effective conductivity of square model with imperfect interface of ellipse Kapitza's type, $\alpha=0.2,{ }^{c_{1}} /{ }_{c_{M}}=10$.

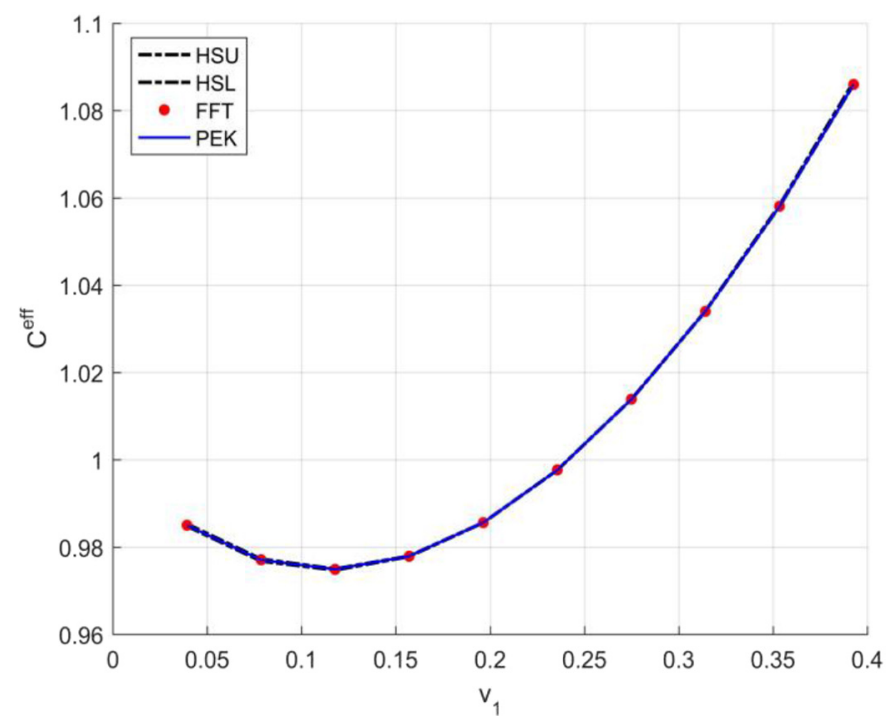

Fig. 7. Effective conductivity of square model with imperfect interface of ellipse Kapitza's type, $\alpha=0.2,{ }^{c_{1}} / c_{c_{M}}=100$.

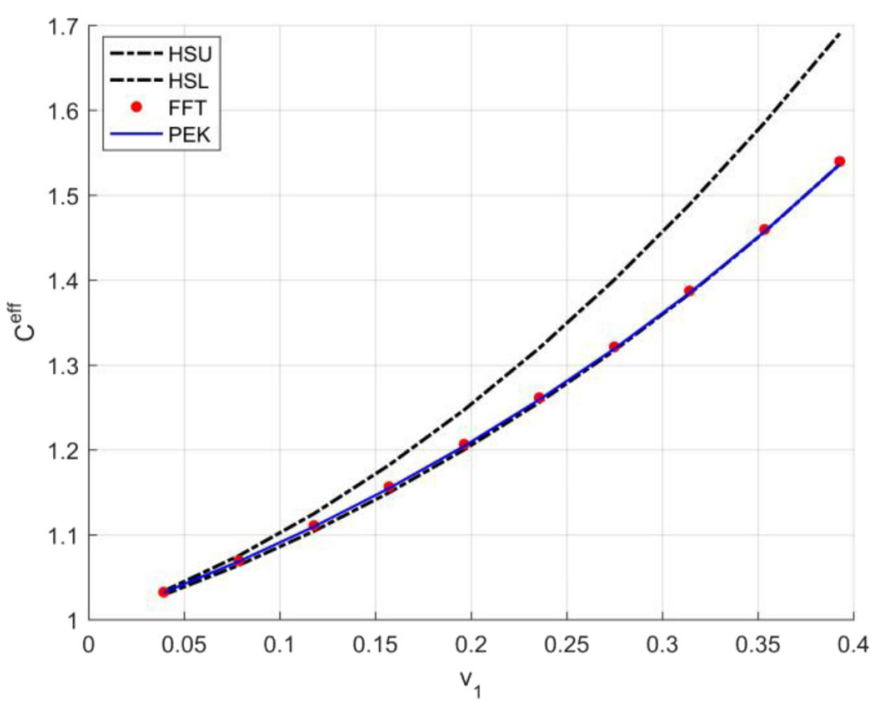

Fig. 8. Effective conductivity of square model with imperfect interface of ellipse Kapitza's type, $\alpha=0.05,{ }^{c_{1}} /{ }_{c_{M}}=10$.

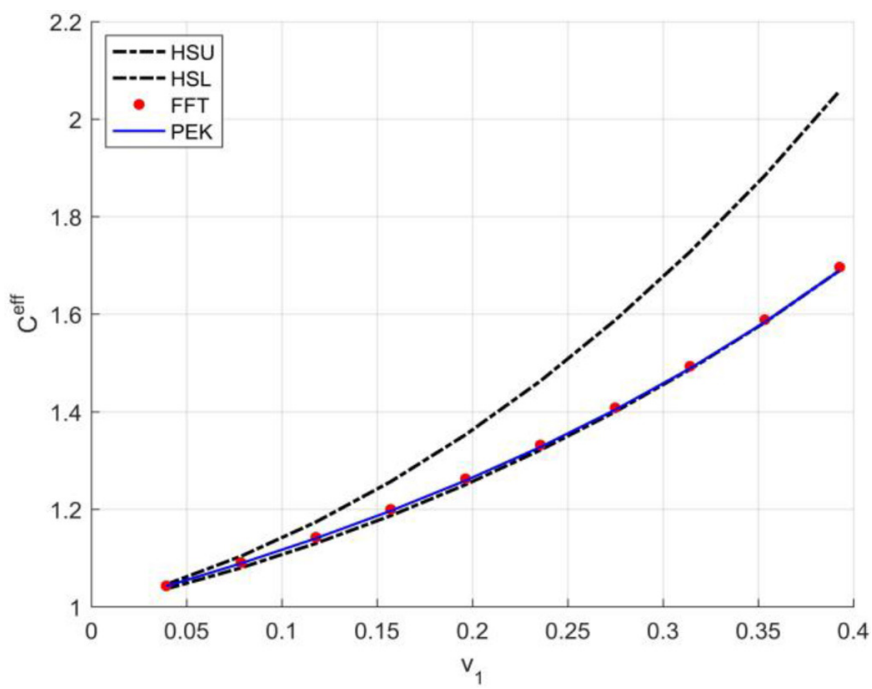

Fig. 9. Effective conductivity of square model with imperfect interface of ellipse Kapitza's type, $\alpha=0.05,{ }^{c_{1}} /{ }_{c_{M}}=100$.

Table 4. Comparison of results $\left(C^{\text {eff }}\right)$ of FFT, PEK and Monchiet's results for square model with one circle inclusion in the unit cell $\alpha=1, a_{1}=a_{2}=R$.

\begin{tabular}{llllllll}
\hline & \multicolumn{3}{c}{$c_{1} / c_{M}=10$} & & \multicolumn{3}{c}{$c_{1} / c_{M}=100$} \\
\cline { 1 - 2 }$v_{1}$ & PEK & FFT & Monchiet [15] & & PEK & FFT & Monchiet [15] \\
\hline 0.0314 & 0.9586 & 0.9588 & 0.9595 & 0.9260 & 0.9589 & 0.9591 & 0.9599 \\
0.0707 & 0.9253 & 0.9255 & 0.9265 & 0.9266 & 0.9275 \\
0.1257 & 0.8942 & 0.8943 & 0.8948 & 0.8974 & 0.8975 & 0.8983 \\
0.1963 & 0.8697 & 0.8698 & 0.8699 & 0.8764 & 0.8765 & 0.8770 \\
0.2827 & 0.8547 & 0.8547 & 0.8548 & 0.8666 & 0.8666 & 0.8670 \\
0.3848 & 0.8511 & 0.8511 & 0.8510 & 0.8705 & 0.8706 & 0.8707 \\
0.5027 & 0.8607 & 0.8607 & 0.8606 & 0.8907 & 0.8907 & 0.8907 \\
0.6362 & 0.8855 & 0.8854 & 0.8852 & 0.9299 & 0.9299 & 0.9297 \\
0.7543 & 0.9179 & 0.9178 & 0.9175 & & 0.9777 & 0.9777 & 0.9775 \\
\hline
\end{tabular}


imperfect interfaces of Kapitza's type while still ensuring accuracy. FFT and PEK give almost same results, the estimation obtained by the FFT and PEK scheme can be viewed an excellent approximation for the effective thermal conductivity of the square models. The FFT results in a special case $r=1, a_{1}=a_{2}=R$ which were compared with results of Monchiet, the results of the comparison are completely close. Especially with the simple expression PEK for coated ellipse aggregate model that can be applied to good results for imperfect interface contact.

\section{References}

1. Z. Hashin, S. Shtrikman, A variational approach to the theory of the elastic behaviour of multiphase materials, J. Mech. Phys. Solids 11, 127-140 (1963)

2. R. Hill, Theory of mechanical properties of fibre-strengthened materials: I. Elastic behavior, J. Mech. Phys. Solids 12, 199-212 (1964)

3. J.R. Willis, Variational and related methods for the overall properties of composite materials, in Advances in Applied Mechanics (Academic Press, New York, 1981)

4. D.C. Pham, L.D. Vu, V.L. Nguyen, Bounds on the ranges of the conductive and elastic properties of randomly inhomogeneous materials, Philos. Mag. 93, 2229-2249 (2013)

5. J.D. Eshelby, The determination of the elastic field of an ellipsoidal inclusion, and related problems, Proc. Roy. Soc. London A 241, 376-396 (1957)

6. S. Torquato, Random Heterogeneous Materials (SpringerVerlag, New York, 2002)

7. R.A. Hill, Self-consistent mechanics of composite materials, J. Mech. Phys. Solids 13, 213-222 (1965)

8. T. Mori, K. Tanaka, Average stress in the matrix and average elastic energy of materials with misfitting inclusions, Acta Metall. 21, 571-574 (1973)

9. T.-K. Nguyen, D.-C. Pham, V.-L. Nguyen, Conduction in 2-D and 3-D dimensional spherically-symmetric anisotropic-coating inclusion composites, Int. J. Eng. Sci. 154, 103352 (2020)

10. J. Michel, H. Moulinec, P. Suquet, Effective properties of composite materials with periodic microstructure: a computational approach, Comput. Methods Appl. Mech. Eng. 172, 109-143 (1999)

11. J. Yvonnet, Q.C. He, Q.Z. Zhu, J.F. Shao, A general and efficient computational procedure for modeling the Kapitza thermal resistance based on XFEM, Comput. Mater. Sci. 50, 1220-1224 (2011)

12. V.-L. Nguyen, FFT and Equivalent-inclusion approach for effective conductivity of three-phase composites with random coated-ellipse inclusion, Eng. Res. Express 3, 025014 (2021)

13. S. Nemat-Nasser, M. Hori, Micromechanics: Overall Properties of Heterogeneous Materials (North-Holland, Amsterdam, 1999)

14. N. Van Luat, N. Trung Kien, FFT-simulations and multicoated inclusion model for macroscopic conductivity of $2 \mathrm{D}$ suspensions of compound inclusions, Vietnam J. Mech. 37, 169-176 (2015)

15. V. Monchiet, FFT based iterative schemes for composites conductors with non-overlapping fibers and Kapitza interface resistance, Int. J. Solids Struct. 135, 14-25 (2018)

16. Y. Benveniste, Model of thin interphases and the effective medium approximation in composite media with curvilinearly anisotropic coated inclusions, Int. J. Eng. Sci. 72, 140-154 (2013)

17. H. Le Quang, D.C. Pham, G. Bonnet, Q.C. He, Estimations of the effective conductivity of anisotropic multiphase composites with imperfect interfaces, Int. J. Heat Mass Transfer 58, 175-187 (2013)

18. H. Le Quang, T.L. Phan, G. Bonnet, Effective thermal conductivity of periodic composites with highly conducting imperfect interfaces, Int. J. Therm. Sci. 50, 1428-1444 (2011)

19. N. Trung Kien, N. Van Luat, P. Duc Chinh, Effective conductivity of isotropic composite with Kapitza thermal resistance, Vietnam J. Mech. 40, 377-386 (2018) 